\title{
Overthinking in Producing Arts and Crafts: A Metacognitive Analysis
}

\author{
Ali Redha Hussain \\ Higher Institute of Dramatic of Arts, Kuwait City, Kuwait \\ Email: dr.ali_uk19@yahoo.com
}

How to cite this paper: Hussain, A. R. (2021). Overthinking in Producing Arts and Crafts: A Metacognitive Analysis. Art and Design Review, 9, 284-290. https://doi.org/10.4236/adr.2021.93023

Received: July 29, 2021

Accepted: August 24, 2021

Published: August 27, 2021

Copyright $\odot 2021$ by author(s) and Scientific Research Publishing Inc. This work is licensed under the Creative Commons Attribution International License (CC BY 4.0).

http://creativecommons.org/licenses/by/4.0/

\begin{abstract}
This study uses the meta-cognitive model (developed in clinical psychology primarily in relation to depressive conditions) to explain the causal dynamics of why artists, in always looking for "perfection", can experience mental states which predicate modes of overthinking which can be detrimental to their careers and/or projects. The meta-cognitive model proposes a specific causal relationship between metacognition and the regulation of thought processes, in which overthinking pertains to excessive metacognitive intervention or total lack thereof. The aim of this study is to establish a practical and implementable meta-cognitive intervention for arts practitioners, arts students, and teachers of arts practice, which will help in avoiding (or teaching how to avoid) the pitfalls of overthinking; to determine a practical "cognitive tool", or mental framework, in the process of making arts. This meta-cognitive tool is intended as an abstract and intellectual complement to the concrete and formal compositional skills of art production. A key focus of this study concerns the liminal points between healthy/productive and unhealthy (and potentially pathological) aspects of creative overthinking, considering how excessive rumination factors into creative traits such as perfectionism and attention to detail in the production of artworks; looking, further, at how overthinking can affect the mind negatively, such as inducing anger and anxiety for example.
\end{abstract}

\section{Keywords}

Meta-Cognitive Model, Metacognition, Overthinking, Arts, Crafts

\section{Introduction}

This paper examines the causes, consequences, and counteractive measures for overthinking in the creative process, thus identifying practical guidelines which allow artists and craftspeople to overcome problems associated with excessive 
rumination-creative, professional, and clinical. According to the meta-cognitive model, environmental stimuli can trigger excessive ruminative processes which, if left unmanaged, can catalyse negative thought patterns; cycles of surfeit internal cogitation that prohibit productive external output-" "paralysis through analysis". This is a serious problem for creative practitioners because it represents a profound hindrance to the productive process; potentially even broaching on pathological behaviours, threatening further harmful (to health) repercussions. The problem is salient and important in the arts and crafts context because the liminal division between diligent, critical attention to detail and process, and unhealthy obsessing and rumination, is blurred and imbricated. For this reason, a pragmatic modus operandi in navigating this treacherous liminal space is of great utility.

\section{Overthinking (Excessive Rumination)}

Overthinking has been defined as an "excessive rumination" or a "loop of unproductive thoughts. Overthinking can also be considered as an excessive amount of thoughts that are unnecessary. Overthinking can be associated with anxiety" (Petric, 2018).

Indeed, much research in this field has been oriented around the prominence and function of overthinking in depression related conditions, with an eye to identifying the causes of excessive rumination thereby to obviate or alleviate them (Sumner, 2012). This is because overthinking is often associated with depressive conditions, where an individual tends to obsess over some (negative or worrisome) idea, leading to exacerbation of the problem. Overthinking is also closely associated with excessive working, and with tasks and processes which demand extreme dedication and focus. There is, then, an ambiguous liminal territory between productive, useful amounts of ample cogitation and unproductive, excessive amounts of same. For artists and craftspeople, being able to negotiate this space, capitalising on the productive elements, while avoiding the unproductive ones, is imperative. The productive elements of deep thinking can be essential in producing complex art works and are regularly associated with ideals of precision artists or craftspeople, the "perfectionist" with exacting attention to detail; are imbued with positive connotations. However, these behavioural traits can easily pass the liminal boundary into cognitive territory with destructive implications, where perfectionism and exactitude summon excessive cognitive patterns. In this instance: "over-thinking and over-working can demonstrate an overuse of cognitive information processing and a lack of faith perceptual (trusting how the [art] looks) or affective (trusting how it feels) processing" (Hinz, 2019). Accordingly, while intense intellectual and emotional effort may well be required for arts and crafts, there is a potential danger if cognition is over-privileged at the expense of other perceptual perspectives. Being able to effectively navigate the liminal space between productive and destructive overthinking is vital; a competence which indeed merits a considered and reflective theoretical perspective. 
The degree to which artists and craftspeople are susceptible to overthinking raises questions about metacognition and how much control individuals command in preventing excessive rumination. Cognitive processes are, at least, in varying degrees, under the artist's control, especially insofar as metacognition is in play. Metacognitive processes are a form of cognitive "self-regulation" concerning one's "capability" to think about one's own "thoughts, feelings and actions"; and hence "by anticipating the outcomes [one's] behaviours will produce and reflecting on previous attempts, people become more or less likely to make plans of action". The authors continue to observe that metacognition factors highly in creative endeavours: "artists and writers have [emphasised] the importance of incubation, or the cessation, of cognitive monitoring" (Alexande et al., 2017). On this analysis, the insights of the meta-cognitive model-that one can identify and regulate one's own thought processes-should provide an excellent foundation for navigating the liminal space between productive and destructive thinking/overthinking.

\section{Unhealthy versus Healthy Rumination}

Almost certainly, there is no objective or unambiguous line between what one may consider productive and destructive thinking/overthinking; for such will be subjectively associated with the particular cognitive profile of the creative person in question. Nevertheless, following the precepts of the meta-cognitive model, there plausibly must be a means of distinguishing when thought processes are devolving in a negative manner. So, when does a productive and "healthy" amount of rumination cross the boundary into counterproductive and "unhealthy" overthinking? The case is complex because the liminal boundaries will not be equivalent between people. Thus one may identify a causal connection between uncertainty (concerning future outcomes) and negative emotional impacts (anxiety). One is, in this case, dealing with anxious pathology deriving from behavioural traits connected to anticipatory cognitive patterns (as defences against a future threat). One can a priori note the intuitive logic of experiencing anxiety over the unknown - as a logical adaptive process, to obviate negative future events/outcomes. This is logical and healthy when applied in proportionate measure the degree of threat. "Fear and anxiety can be distinguished according to how much certainty one has regarding the likelihood, timing, or nature of future threat"; and anxiety thus entails "cognitive, and behavioral changes in response to uncertainty about potential future threat" (Connors \& Levy, 2020). The danger, for artists and craftspeople, is where these defensive measures degenerate into a maladaptive behaviour, where anxiety-yielding ruminations are pursued in excess. The meta-cognitive model provides a variety of strategies for preventing or overcoming anxiety. Of key importance is to interrupt destructive thought patterns, which become self-perpetuating and mutually reinforcing. For example, the "belief that worrying is uncontrollable leads to a persistence of worrying" exactly because the "person does not use their mind to interrupt the 
process". In the metacognitive model, the emphasis is on "controlling attention and regulating excessive thinking such as worry", processes which are "directly linked to underlying metacognitive beliefs" (Nordahl \& Wells, 2017). In the context of artists, such thought patterns threaten destructive psycho-emotional byproducts, like frustration, anger, fear and despair, which may inhibit the creative process. As a result, it is important that effective interventions are introduced to preclude these negative outcomes. One suggested intervention, which was found to have empirical validity, concerns managing one's meta-beliefs, or one's thoughts about one's own thought patterns (Wells \& Papageorgiou, 2001). For instance, certain beliefs that anxiety and worry cannot be controlled at all can be brought into focus. In this form of metacognitive intervention, the subject focuses on adapting their meta-beliefs, to better manage their metacognition (Nordahl et al., 2017). Accepting that such control is possible, then, is a significant element of the process.

\section{Metacognition as Intervention}

The meta-cognitive model is relevant to arts/crafts because it allows for a practical, outcome-oriented modality, whereupon "clarifying the [meta-cognitive] construct" can serve as a "precursor for developing more targeted and effective interventions" (Petrini \& Arendt-Nielsen, 2020). Targeted approaches can employ "discipline-specific design" where "educators prompt defensive posture on the need to avoid overthinking, analysis-paralysis, and the like" (Folkmann et al., 2019). The pedagogical crux of the meta-cognitive process is to impart to students (and practitioners) a set of foundational meta-beliefs (Barnett \& Davies, 2015). There are as follows: 1) the belief that thought can be managed and controlled; 2) that negatives thoughts can be therefore regulated and contained; 3) that some unpleasant emotions (like anxiety) can be viewed in a positive light and used as cues to change course (Crombez et al., 2020). These three pillars frame healthy adaptive interventions.

Practically, in arts/crafts, one would frame the meta-belief that meta-cognition is an inevitable part of the process, needing to be nurtured and developed. Barnett gives the example of how anxiety can be leveraged to the artist's advantage, if appropriately managed, using "creativity and problem-solving heuristics", interpreting anxiety as an early-warning sign. Here, the artist can early-on detect negative traits surfacing, thus "head off" negative thought cycles (which, left to exacerbate, would become inhibitive); in essence, enabling a "strategy for improving self-control" through reflective awareness of cognitive processes (Crombez et al., 2020). These self-control mechanisms, or "interventions", could be incorporated into the broader framework of the creative process and taught to arts and crafts students accordingly (Day et al., 2019).

Arguably the key "intervention" would be to distinguish between productive/destructive modes of deep thinking, so not to "throw the baby out with the bathwater" (Morrison, 2020). As Morrison notes, while "analysing or over- 
thinking" a work of art "can disrupt the flow of inspiration", an "overt foregrounding of technique can actually unlock new possibilities and fresh innovation, revealing new strategies and truth" (Kershaw \& Wade, 2012).

In identifying the liminal boundary between productive/destructive cogitation, artists (following Morrison's insight) could leverage meta-cognitive awareness into meta-cognitive tools: context-specific interventions. Feelings of anxiety potentially flag the onset of excessive rumination, providing the cue for the artist to employ further meta-cognitive perspectives. For instance, the artist could reflect on the cause of the anxiety, in order to locate thereby an apt corrective. Take the example of fear (of failure). "Fear of failure and overthinking a situation interfere with performance and block access to creativity. If an individual begins to ruminate over all the possible things that might go wrong, anxiety is raised, and the ability to access the zone is compromised" (Lefebvre \& Jensen, 2019). An artist detecting this root cause for excessive rumination could consequently rationalise that such fear will constitute a self-fulfilling prophesy if not contained-the fear will eventuate the failure (Zohreh \& Ghazal, 2018). One logical solution for the artist on this occasion would be to focus on the process and not the (merits of) outcome, thus to keep working and stop ruminating (Fernie et al., 2015). This is not a one-size-fits-all solution, of course; nor is it intended to be. The intervention deployed will depend upon the specific needs or inhibitions of the artist at that time. The key is the process (of meta-cognition) used as a tool-and not the specific thought cycle that tool is used to address. Process is top priority, using the meta-cognitive model to direct appropriate interventions; it is a cognitive framework which helps in structuring thought, one based on the meta-belief that thoughts can be regulated and directed away from negative and toward positive patterns. This is an essential finding of this paper, that (following the meta-cognitive model) thought necessarily can be brought under control and regulated, as it were, autonomously by the individual. Metacognition, on this analysis, functions as an early warning system for potentially problematic thought patterns which, in unregulated, could lead to negative psychological outcomes (Adams \& Turk, 2015). One can use this early warning to obviate or mitigate overthinking. At the core of this process is the establishment of meta-beliefs, which assert that thoughts can indeed be managed, and thereby the subject is not at the mercy of worry and anxiety; but, instead, they have agency in their own cognitive profile (Fisher \& Wells, 2009). Applying this to the arts and crafts production context, it would be advantageous to expand the conceptual purview of how metacognition factors into the logic of artistic creation-not as some external theoretical model, but as a central component, which needs to be considered, if not continually, at least regularly.

\section{Conclusion}

The meta-cognitive model is potentially a very powerful tool for empowering artists and craftspeople with agency, specifically via the meta-belief that thought 
may be regulated. This meta-belief supports a powerful paradigm shift, where the artist is not a passive object of their thought patterns, but rather an active agent in directing cognition. By adopting the assumptions of the metacognitive model, creative practitioners should be able to limit the potential for destructive overthinking, through a reflexive and critical awareness of their own thought patterns. Hence the meta-cognitive model can be adapted as a tool to recognise and overcome the early signs of excessive rumination when they initially manifest.

\section{Conflicts of Interest}

The author declares no conflicts of interest regarding the publication of this paper.

\section{References}

Adams, L. M., \& Turk, D. C. (2015). Psychosocial Factors and Central Sensitivity Syndromes. Current Rheumatology Reviews, 11, 96-108. https://doi.org/10.2174/1573397111666150619095330

Alexande, P. A., Schunk, D. H., \& Greene, J. A. (2017). Handbook of Self-Regulation of Learning and Performance (p. 17). Taylor \& Francis. https://doi.org/10.4324/9781315697048

Barnett, R., \& Davies, M. (2015). The Palgrave Handbook of Critical Thinking in Higher Education. Palgrave Macmillan.

Connors, J., \& Levy, J. (2020). COVID-19 and Its Implications for Thrombosis and Anticoagulation. Blood, 135, 2033-2040. https://doi.org/10.1182/blood.2020006000

Crombez G., Paepe, A. L. D., Veirman, E., Eccleston, C., Verleysen, G., \& Van Ryckeghem, D. M. L. (2020). Let's Talk about Pain Catastrophizing Measures: An Item Content Analysis. PeerJ, 8, Article No. e8643. https://doi.org/10.7717/peerj.8643

Day, M. A., Matthews, N., Newman, A., Mattingley J. B., \& Jensen, M. P. (2019). An Evaluation of the Behavioral Inhibition and Behavioral Activation System (BIS-BAS) Model of Pain. Rehabilitation Psychology, 64, 279-287. https://doi.apa.org/doi/10.1037/rep0000274

Fernie, B., Maher-Edwards, L., Murphy, G., Nikčević, A. V., \& Spada, M. M. (2015). The Metacognitions about Symptoms Control Scale: Development and Concurrent Validity. Clinical Psychology \& Psychotherapy, 22, 443-449. https://doi.org/10.1002/cpp.1906

Fisher, P. L., \& Wells, A. (2009). Metacognitive Therapy: Distinctive Features. Routledge. https://doi.org/10.4324/9780203881477

Folkmann, M. N, Skou, P. S., Jensen, H-S., \& Munch, V. S. (2019). Design Culture Objects and Approaches (p. 218). Bloomsbury Publishing.

Hinz, L. D. (2019). Expressive Therapies Continuum: A Framework for Using Art in Therapy (p. 169). Taylor \& Francis. https://doi.org/10.4324/9780429299339

Kershaw, C. J., \& Wade, W. (2012). Brain Change Therapy: Clinical Interventions for SelfTransformation (p. 280). W. W. Norton.

Lefebvre, J. C., \& Jensen, M. P. (2019). The Relationships between Worry, Happiness and Pain Catastrophizing in the Experience of Acute Pain. European Journal of Pain, 23, 1358-1367. https://doi.org/10.1002/ejp.1405

Morrison, S. A. (2020). Dancefloor-Driven Literature: The Rave Scene in Fiction. Blooms- 
bury. https://doi.org/10.5040/9781501357701

Nordahl, H., \& Wells, W. (2017). Testing the Metacognitive Model against the Benchmark CBT Model of Social Anxiety Disorder: Is It Time to Move Beyond Cognition? PLoS ONE, 12, e0177109. https://doi.org/10.1371/journal.pone.0177109

Nordahl, H., Nordahl, H. M., Hjemdal, O., \& Wells, A. (2017). Cognitive and Metacognitive Predictors of Symptom Improvement Following Treatment for Social Anxiety Disorder. Clinical Psychology \& Psychotherapy, 24, 1221-1227.

https://doi.org/10.1002/cpp.2083

Petric, D. (2018). Emotional Knots and Overthinking. Research Gate.

Petrini, L., \& Arendt-Nielsen, L. (2020). Understanding Pain Catastrophizing: Putting Pieces Together. Frontiers in Psychology, 11, Article No. 603420. https://doi.org/10.3389/fpsyg.2020.603420

Sumner, J. A. (2012). The Mechanisms Underlying over General Autobiographical Memory: An Evaluate Review of Evidence for the CaR-FA-X Model. Clinical Psychology Review, 32, 34-48. https://doi.org/10.1016/j.cpr.2011.10.003

Wells, A., \& Papageorgiou, C. (2001). Brief Cognitive Therapy for Social Phobia: A Case Series. Behaviour Research and Therapy, 39, 713-720. https://doi.org/10.1016/S0005-7967(00)00036-X

Zohreh, A., \& Ghazal Z. (2018). Behavioral Systems and Difficulty with Emotional Regulation Predict Adolescents' Addiction potential. Journal of Child \& Adolescent Substance Abuse, 27, 272-276. https://doi.org/10.1080/1067828X.2018.1474824 